\title{
On the Nature of the Born Probabilities
}

\author{
Andreas Schlatter \\ Burghaldeweg 2F, Küttigen, Switzerland \\ Email:schlatter.a@bluewin.ch
}

How to cite this paper: Schlatter, A. (2017) On the Nature of the Born Probabilities. Journal of Modern Physics, 8, 756760.

https://doi.org/10.4236/jmp.2017.85047

Received: March 13, 2017

Accepted: April 14, 2017

Published: April 17, 2017

Copyright $\odot 2017$ by author and Scientific Research Publishing Inc. This work is licensed under the Creative Commons Attribution International License (CC BY 4.0).

http://creativecommons.org/licenses/by/4.0/

\begin{abstract}
The Born-rule, which assigns probabilities $p_{a}=\frac{\left|\psi_{a}\right|^{2}}{\|\psi\|^{2}}$ to measurement outcomes, is one of the fundamental axioms of quantum physics. It dates back to the time of the establishment of the formalism of quantum physics in the first half of the $20^{\text {th }}$ century. From the beginning, and particularly in connection with the development of different interpretations of the theory, there has been a desire/need to better understand the true nature of the Born-probabilities. Are they classical/epistemic of origin or are they irreducible and of on tic stature as a kind of intrinsic propensities of physical systems? We show that, by only using the mathematical formalism of the original theory, we find a possible answer.
\end{abstract}

\section{Keywords}

Quantum Measurement, Quantum Ontology, Density Operator

\section{Introduction}

The formalism of quantum physics has been developed during the first decades of the $20^{\text {th }}$ century. It describes a physical system as an element $|\psi\rangle$ of some appropriate Hilbert space $H$ and physical, observable quantities as eigenvalues $a \in \mathbb{R}$ in the spectrum $\sigma_{A} \subseteq \mathbb{R}$ of self-adjoint operators $A \in L(H)$ on that Hilbert space. The eigenvalue-eigenstate postulate says that, whenever a system is found to have a value $a \in \sigma_{A}$, then it is in the corresponding eigenstate $\left|e_{a}\right\rangle$ of $A$. Inversely, if a system $|\psi\rangle$ is represented in the eigenbasis, $\left\{\left|e_{a}\right\rangle\right\}_{a \in \sigma_{A}}$, of $A,|\psi\rangle=\sum_{a \epsilon \sigma_{A}} \psi_{a}\left|e_{a}\right\rangle, \psi_{a} \epsilon \mathbb{C}$, then the system is experimentally found to have eigenvalue $a$ with probability

$$
p_{a}=\left|\psi_{a}\right|^{2} /\|\psi\|^{2} .
$$

This is the Born-rule [1]. The Born-rule together with the eigenvalue-eigenstate link constitutes the measurement postulate. No violation of the Born-rule 
has ever been discovered experimentally. The measurement postulate is incompatible with a further postulate of quantum mechanics, namely the unitary evolution of the quantum state. Ever since the establishment of the theory there have been different interpretations and extensions of quantum physics in order to solve this incompatibility, known as the "measurement problem".

There are some questions, which naturally arise with regard to the Born-rule. Firstly, why are there probabilities in the first place and secondly, what kind of probabilities are they? Both questions are intimately linked to interpretations of quantum mechanics and have in this context found various answers. Focusing on the second question we find the opinions, starting on the realist side, that the probabilities might be objective, irreducible properties of quantum systems, as in the GRW interpretations [2] or, passing to the epistemic/instrumentalist side, subjective degrees of belief [3] or, yet represent something else, like rational preferences in the decision-theoretic explanations of the many-worlds interpretation [4] [5] [6] [7]. Because the formalism of quantum physics shows the kind of ontological under determination it does, the Born-rule does as well. In this paper we follow the original, basic formalism and will give a possible answer to the question of the nature of the probabilities.

\section{Some Formalism}

Given the resolution of a state $|\psi\rangle \epsilon H$ in the eigenbasis $\left|e_{a}\right\rangle, a \epsilon \sigma_{A}$, of an operator $A,|\psi\rangle=\sum_{a \in \sigma_{A}} \psi_{a}\left|e_{a}\right\rangle, \psi_{a} \in \mathbb{C}$, we can form the corresponding density matrix $\varrho_{\psi}:=|\psi\rangle\langle\psi|$ with matrix-entries $\left(\varrho_{\psi}\right)_{a a^{\prime}}=\psi_{a} \psi_{a^{\prime}}^{*}$. This matrix is a self-adjoint operator, a projection operator in this case, satisfying $\varrho_{\psi}^{2}=\varrho_{\psi}$. If $\psi$ is normalized, $\|\psi\|=1$, then so is the trace of $\varrho_{\psi}, \operatorname{tr}\left(\varrho_{\psi}\right)=1$, and the diagonal matrix-elements of $\varrho_{\psi}$ happen to correspond to the numerical values in the Born probabilities $\left(\varrho_{\psi}\right)_{a a}=\left|\psi_{a}\right|^{2} /\|\psi\|^{2}$. By the correspondence, $|\psi\rangle \rightarrow \varrho_{\psi}$, there is an alternative formulation of the postulates of traditional quantum physics in terms of density operators. If a density operator satisfies $\varrho_{\psi}^{2}=\varrho_{\psi}$, i.e. is a projector, we say that $\varrho_{\psi}$ is pure. Density operators can be thought to incorporate the known information about a state and this allows a generalization. Given a set $\left\{\varrho_{a}:=\left|e_{a}\right\rangle\left\langle e_{a}\right|\right\}_{a \in \sigma_{A}}$ of pure density operators and a set of probability weights $\left\{p_{a}\right\}_{a \in \sigma_{A}}, \sum_{a \epsilon \sigma_{A}} p_{a}=1$, we can form a new density operator

$$
\varrho:=\Sigma_{a \epsilon \sigma_{A}} p_{a} \varrho_{a} \text {. }
$$

We say that the operator $\varrho$ in (2) is a mixed state. Mixed states $\varrho$ are formally self-adjoint operators with $\operatorname{tr}(\varrho)=1$ but no projectors, so $\varrho^{2} \neq \varrho$. The interpretation of mixed states is entirely classical in the sense that the probabilities $\left\{p_{a}\right\}_{a \in \sigma_{A}}$ reflect a lack of knowledge and hence belief in the likelihood of elements of a set of possible preparations, done in a lab for instance. There is a theorem due to Gleason [8], which basically says that the trace-function $\varrho \rightarrow \operatorname{tr}(\varrho A)$ is the unique probability measure, which is faithful to the postulates of quantum physics on Hilbert space ${ }^{1}$. Gleason's theorem tells us that we

${ }^{1}$ Under condition that the dimension of the Hilbert space is at least three. 
are looking at the right probabilities. But it is per se not helpful to better understand the nature of the Born-probabilities.

\section{Measurement}

Assume there is a density matrix $\varrho$ and basis (eigen)-states $\left|e_{a}\right\rangle, a \epsilon \sigma_{A}$, corresponding to some self-adjoint operator $A \epsilon L(H)$. Assume in addition that there is an additional system $\mathcal{E}$ with orthonormal basis states $\{|n\rangle\}_{n \leq N}$, which we assume originally to be in the base state $\varrho_{0}=|0\rangle\langle 0|$. A measurement of $\varrho$ by the probe $\mathcal{E}$ is an operation $U$ on the joint system $\varrho_{\text {joint }}=|0\rangle\langle 0| \otimes \varrho$

$$
U(|0\rangle\langle 0| \otimes \varrho) U^{*},
$$

where $U$ is unitary $U U^{*}=\mathrm{II}^{2}$. A general unitary transformation on a tensor-product, expressed in the respective bases, can be written as a matrix

$$
\begin{aligned}
U & =\sum_{a n, a^{\prime} n^{\prime}} u_{a n, a^{\prime} n^{\prime}}|a\rangle|n\rangle\left\langle a^{\prime}\right|\left\langle n^{\prime}\right| \\
& =\sum_{n n^{\prime}} A_{n n^{\prime}} \otimes|n\rangle\left\langle n^{\prime}\right|,
\end{aligned}
$$

where the operators $A_{n n^{\prime}}$ are given by $A_{n n^{\prime}}=\sum_{a a^{\prime}} u_{n a, n^{\prime} a^{\prime}}|a\rangle\left\langle a^{\prime}\right|$. We denote the diagonal sub-block $A_{n 0}$ simply by $A_{n}$. Since $U$ is unitary, we have

$$
\left\langle 0\left|U U^{*}\right| 0\right\rangle=\sum_{n} A_{n} A_{n}^{*}=\text { II. }
$$

Conversely, we can choose any set of operators $A_{n}$ satisfying the resolution of the identity-condition (5) to define a measurement on an initial joint state $\varrho_{\text {joint }}=|0\rangle\langle 0| \otimes \varrho$. We now have the necessary elements in place to give the main argument.

\section{The Born-Rule}

Assume there is a quantum system $\mathcal{S}$ in a, not necessarily normalized, pure state $\psi \epsilon H$ with representation $\psi=\sum_{a \leq M} \psi_{a}\left|e_{a}\right\rangle, \psi_{a} \epsilon \mathbb{C}$, and corresponding density matrix $\varrho_{\psi}=|\psi\rangle\langle\psi|$ with matrix elements

$(\varrho)_{a, a^{\prime}}=\left(\psi_{a} \psi_{a^{\prime}}^{*}\right), 1 \leq a^{\prime}, a \leq M$. We further assume all the postulates of traditional quantum physics as above, except the Born-rule, and ask ourselves where the probabilities come from.

Assume there is a second system $\mathcal{E}$ with basis $\{|n\rangle\}_{n \leqq N}$ and an observer who would like to know in what state $\varrho_{a}=\left|e_{a}\right\rangle\left\langle e_{a}\right|$ the system $\mathcal{S}$ is in, by making an appropriate measurement $U$ on the joint system $\varrho_{\text {joint }}=|0\rangle\langle 0| \otimes \varrho$. If that is possible in the first place, then, having no additional knowledge, the observer does a priori not know in what state $|n\rangle, n \leq N$, the probe will be after the measurement and before observation.

Let the underlying pure state $|\psi\rangle \epsilon H$ have coefficients $\psi_{a}=\sqrt{m_{a}} e^{i \varphi_{a}}, m_{a} \epsilon \mathbb{N}, \varphi_{a} \in \mathbb{R}^{3}$. The probe $\mathcal{E}$ can be chosen appropriately coarsegrained $^{4}$ such that $N=\sum_{a \leq M} m_{a}$ We now introduce probabilities by Laplace's principle of indifference. The observer is after the measurement and before ob-

${ }^{2}$ This follows from the fact that a general interaction evolution $U(t)=e^{-(i / t) H t}$ is unitary.

${ }^{3}$ Since the rational numbers $\mathbb{Q}$ are dense in $\mathbb{R}$, the choice of $m_{a} \epsilon \mathbb{N}$ is general enough.

${ }^{4}$ This coarse-graining is used first by [6] and then by [7] in the context of many-worlds. 
servation in a situation where, by lack of further information, she will a priori attribute to each outcome $\left\langle n\left|U(|0\rangle\langle 0| \otimes \varrho) U^{*}\right| n\right\rangle$ equal probability $p_{n}=1 / N, n \leq N$. This attribution is equivalent to maximizing the entropy function $H(p)=-\sum_{n=1}^{N} p_{n} \log p_{n}$. The observer can therefore write down in the spirit of (2) an average of outcomes

$$
\tilde{\varrho}:=\sum_{n \leq N} 1 / N\left(\left\langle n\left|U(|0\rangle\langle 0| \otimes \varrho) U^{*}\right| n\right\rangle\right)=\sum_{n \leq N} 1 / N\left(A_{n} \varrho A_{n}^{*}\right) .
$$

For our purpose we now chose the operators $A_{n}$ to be the scaled projectors $\left\{\tilde{P}_{a_{k}}:=\left(1 / \sqrt{m_{a}}\right) P_{a}\right\}_{a \leq M, k \leq m_{a}}$ to the basis-states $\left|e_{a}\right\rangle, a \leq M$. Note that we have replaced the simple-index $n$ by the double-index $a_{k}$. This choice is consistent with the demands of a measurement, since the $\tilde{P}_{a_{k}}$ satisfy (5)

$$
\sum_{n \leq N} \tilde{P}_{n}^{*} \tilde{P}_{n}=\sum_{a \leq M} \sum_{k \leq m_{a}} \tilde{P}_{a_{k}}^{*} \tilde{P}_{a_{k}}=\sum_{a \leq M} P_{a}^{*} P_{a}=\text { II. }
$$

Therefore we can write (6) in the following form

$$
\begin{aligned}
\tilde{\varrho} & =\sum_{n \leq N} 1 / N\left(\tilde{P}_{n} \varrho \tilde{P}_{n}^{*}\right)=\sum_{a \leq M} \sum_{k \leq m_{a}} 1 / N\left(\tilde{P}_{a_{k}}^{*} \varrho \tilde{P}_{a_{k}}\right) \\
& =\sum_{a \leq M} 1 / N\left(P_{a} \varrho P_{a}^{*}\right)=\sum_{a \leq M}\left(m_{a} / N\right) \varrho_{a} .
\end{aligned}
$$

Comparing Equation (8) with Equation (2), we see that $\tilde{\varrho}$ can be viewed as a mixed state with probabilities

which is the Born-rule.

$$
p_{a}=m_{a} / N=\left|\psi_{a}\right|^{2} /\|\psi\|^{2},
$$

\section{Conclusions}

We have in the above derivation not made use of any specific interpretation of quantum mechanics, but relied on two basic assumptions only. The first one is the formalism of density operators and generalized measurement with classical or epistemic probabilities arising in mixed states (2). The second one is Laplace's principle of indifference in order to introduce the concept of probabilities and to assign concrete probability-values $p_{n}=1 / N, n \leq N$, to the mixed state in (6). This is the important step, which helps to avoid the kind of tautological argument based on the reduced density matrix and Gleason's theorem. It bases on a kind of symmetry of the probe states $|n\rangle, n \leq N$, due to a lack of knowledge before observation.

We have found that, given any not necessarily normalized pure state, it is possible to define an observer with an appropriately coarse-grained probe-system ${ }^{5}$ who, by lack of further knowledge, will assign exactly the Born-probabilities, as classical probabilities in the sense of (2), to finding the system in one of the basis-states, after the measurement and before observation. In other words, there is the possibility to interpret the normalized amplitudes of an arbitrary state $\psi \epsilon \mathrm{H}$ as epistemic probabilities for different possible measurement outcomes.

If a quantum state $\psi$ is given to us a priori, then it seems that the Bornprobabilities are objectively given with it and there might be a reluctance to embrace Laplace's principle as fundamental [9]. It seems to us that we can accept ${ }^{5}$ The environment could serve as a probe-system for instance. 
the principle as deeply rooted in our intuition and therefore to be a first principle. Authors, concerned with the many worlds interpretation in [6] [7] for instance, try to give more objective physical justifications for Laplace's principle, mainly by describing the symmetry, which it bases on, in more physical terms. We are not sure that these ideas are really more fundamental or whether they are not the same intuition vested in different garments. We should in all this always remember that quantum states are practically given to us by making preparations, i.e. by correlating them with other systems in laboratories. It is a wonderful fact, however, that the Born-probabilities are confirmed by the corresponding frequencies, if repeated experiments are being done. This would probably not be further impressive, if quantum states would just be results of gathering measurement information. But they can also arise from an initial state by Schrödinger evolution. We at least can say that nature seems to "play the game".

\section{References}

[1] Born, M. (1926) Zeitschriftfür Physik, 37, 863-867.

[2] Ghirardi, G.C., Rimini, A. and Weber, T. (1986) Physical Review D, 34, 470.

[3] Mermin, N.D. (2014) Nature, 507, 421-423.

[4] Deutsch, D. (1999) Proceedings of the Royal Society of London A, 455, 3129-3137. http://www.arxiv.org/abs/quant-ph/9906015

[5] Wallace, D. (2003) Studies in History and Philosophy of Modern Physics, 34, 415-438. http://arxiv.org/abs/quant-ph/0303050 http://philsci-archive.pitt.edu/archive/00001030

[6] Zurek, W.H. (2003) Physical Review Letters, 90, 12.

[7] Carroll, S.M. and Sebens, C.T. (2014) Many Worlds, the Born Rule, and Self-Locating Uncertainty. In: Struppa D. and Tollaksen J., Eds., Quantum Theory. A Two-Time Success Story. Springer, Milano, 157-169.

[8] Gleason, A.M. (1957) Journal of Mathematics and Mechanics, 6, 885.

[9] Zurek, W.H. (2010) Quantum Jumps, Born's Rule, and Objective Reality. In: Many Worlds? Everett, Quantum Theory, \& Reality, Oxford University Press, Oxford, 409. 
Submit or recommend next manuscript to SCIRP and we will provide best service for you:

Accepting pre-submission inquiries through Email, Facebook, LinkedIn, Twitter, etc. A wide selection of journals (inclusive of 9 subjects, more than 200 journals)

Providing 24-hour high-quality service

User-friendly online submission system

Fair and swift peer-review system

Efficient typesetting and proofreading procedure

Display of the result of downloads and visits, as well as the number of cited articles Maximum dissemination of your research work

Submit your manuscript at: http://papersubmission.scirp.org/

Or contact jmp@scirp.org 